\title{
Parapharyngeal Abscess Two Years After Elective Tonsillectomy
}

\author{
Fabio Arciello ${ }^{\mathrm{a}, \mathrm{b}}$, Erika Crosetti ${ }^{\mathrm{a}}$, Diego Di Lisi ${ }^{\mathrm{a}}$, Giovanni Succo ${ }^{\mathrm{a}}$
}

\begin{abstract}
Parapharyngeal abscesses are the second most common site of deep neck infections, and should not be underestimated due to potentially fatal complications. A case of parapharyngeal abscess in a young patient, with a previous history of elective tonsillectomy performed 2 years before, is reported.
\end{abstract}

Keywords: Parapharyngeal abscesses; Tonsillectomy; Deep neck infections

\section{Introduction}

Parapharyngeal abscesses are the second most common sites of deep neck infections, after those arising in the peritonsillar area, of which they are often a consequence [1]. Despite the widespread use of antibiotics in the therapy of head and neck infections, these deep abscesses are still relatively common and should not be underestimated due to potentially fatal complications. A case of parapharyngeal abscess in a young patient, with a previous history of elective tonsillectomy performed 2 years before, is reported.

\section{Case Report}

A 19-year-old male presented to our department referring a 1-year-long history of recurrent fever associated with weakness and elevated white blood cell count. Physical examination revealed mild neck swelling and tenderness, but no

Manuscript accepted for publication March 31, 2014

${ }^{a}$ University Department of Otorhinolaringology, San Luigi Gonzaga, Martini Hospital, Torino, Italy

${ }^{\mathrm{b}}$ Corresponding author: Fabio Arciello, Department of

Otorhinolaringology, Martini Hospital, Via Tofane 71, Torino, Italy.

Email: dott.arciello@gmail.com

doi: http://dx.doi.org/10.14740/jmc1760w other systemic symptoms. His medical history was unremarkable, except for an elective tonsillectomy performed 2 years earlier (at the age of 17) due to recurrent tonsillitis and peritonsillar abscess in the left tonsil. In the suspicion of a hematological malignancy, he was also referred for a complete blood analysis. At the time of last observation, MRI of the cervical region revealed a prestyloid parapharyngeal abscess on the left side (Fig. 1a, b). Direct inspection of the oral cavity did not reveal evident signs of involvement in the acute process documented by MRI, but did reveal fusion of the left palatine pillars (Fig. 2), which was probably a post-tonsillectomy sequela. Explorative fine needle aspiration was performed transorally, and the material was sent for microbiologic culture that subsequently revealed a polymicrobial infection.

Intravenous therapy based on steroids and antibiotics was administered for 10 days. Due to persistence of the abscess and new episodes of high fever (up to $41^{\circ} \mathrm{C}$ ), we performed surgical marsupialization (Fig. 3).

Surgical drainage was promptly performed using an intra-oral approach with healing as second intention. The surgical specimen was sent for both microbiological and pathological analyses. Pathology examination described a chronic gigantocellular infiltrate that was most likely a foreign body reaction to the previous tonsillectomy. Microbiological culture confirmed the polymicrobial population found in the first fine needle aspiration. During hospitalization, the patient continued antibiotic therapy, and the surgical wound was strictly monitored and thoroughly cleansed after every meal. The patient was discharged 8 days after surgery and is now physically well, afebrile, with no evidence of recurrence or sequelae on radiological follow-up (Fig. 4).

\section{Discussion}

Parapharyngeal abscess are the second most common deep neck infection, after those arising in the peritonsillar area, of which they are often a consequence [1]. Anatomically, the parapharyngeal region is located in the upper neck, extending from the skull base to the hyoid bone, and divided by the styloid process and muscles in a prestyloid and postyloid 

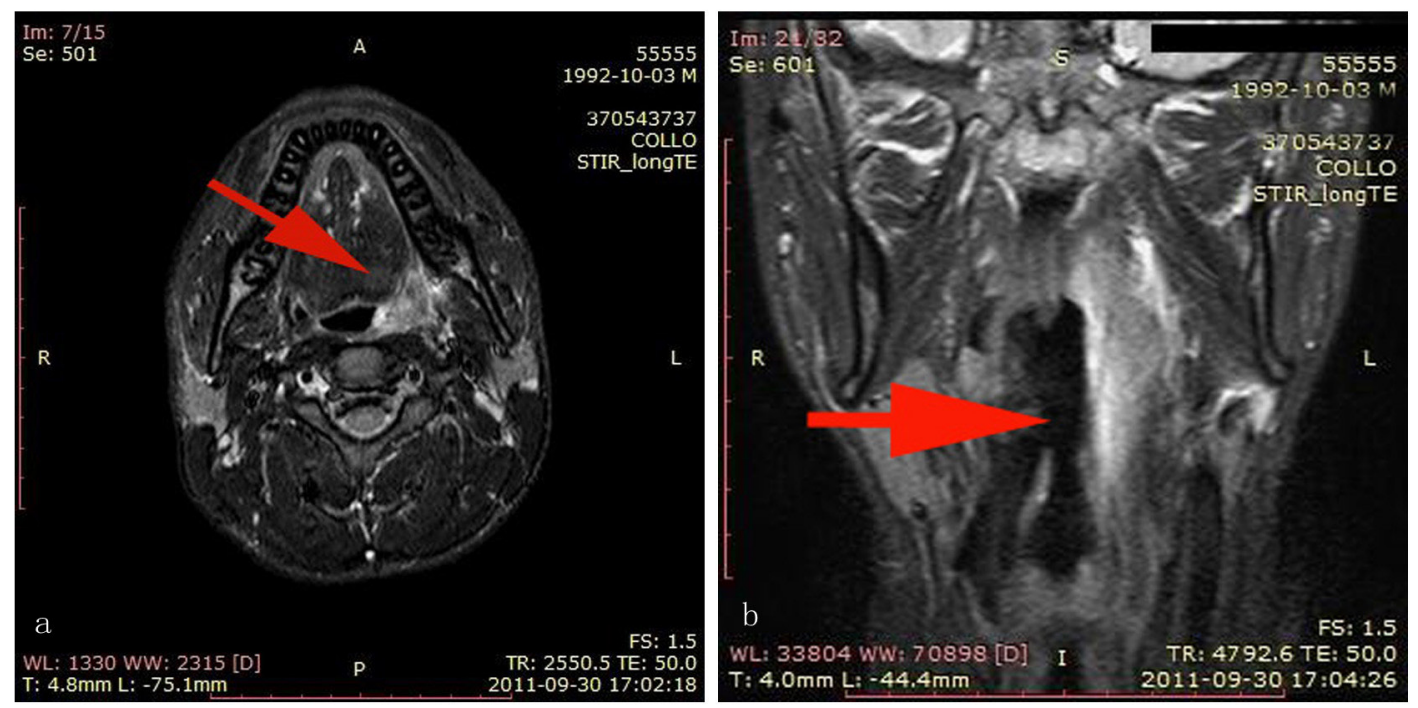

Figure 1. $(a, b)$ MRI of the cervical region revealed a prestyloid parapharyngeal abscess on the left side (arrows).

compartment, the latter containing the carotid sheath.

Clinically, parapharyngeal abscesses can be mistaken for simple quinsy, since both present with fever, trismus and medial displacement of the palatine tonsil, even though frank paraphayngeal abscesses often cause more severe symptoms, such as laryngeal deviation and unilateral neck swelling [2]. In our case, symptoms were unspecific and could be initially misinterpreted as a hematological malignancy. However, MRI revealed inflammatory involvement of the cervical region and documented its extension to the parapharyngeal space, prompting a surgical approach. Parapharyngeal abscess are most commonly diagnosed using contrastenhanced computed tomography, since it allows reliable assessment of the extension of the abscess [3].

Even if some recent reports $[1,4,5]$ have confirmed that intravenous antibiotic therapy alone, associated with strict

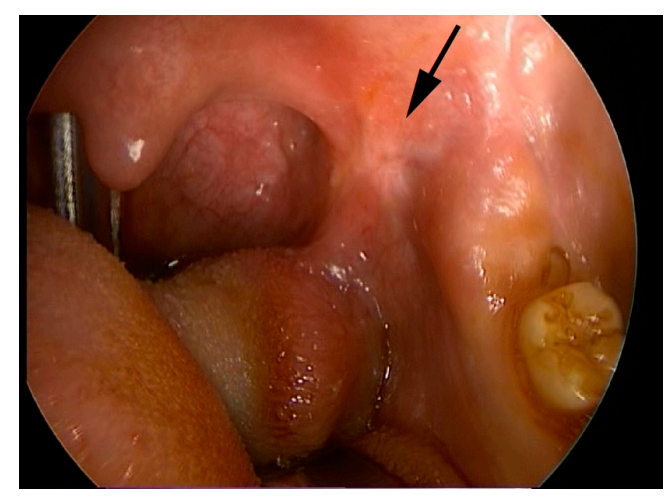

Figure 2. Direct inspection of the oral cavity revealed fusion of the palatine pillars (arrow) without evident signs of involvement in the acute process documented by MRI. radiological follow-up, can be successfully used in the management of uncomplicated parapharyngeal abscess, surgical drainage is the treatment of choice in cases where patients are not responsive to first-line antibiotics, with systemic symptoms or with severe complications, such as airway compromise, meningitis, mediastinitis (through retropharyngeal space) and Lemierre's syndrome [6, 7]. Following these indications, we decided to promptly perform intraoral drainage in our patient, since he did not respond to the antibiotic therapy, and had episodes of high fever. We cannot exclude that the healing wound that created a fusion of the palatine pillars played a role in the pathogenesis of the abscess, and might have concurred in the abscess formation acting as a predisposing factor.

In conclusion, we confirm the importance of early diagnosis of parapharyngeal abscess in order to avoid possible life-threatening complications, and highlight how this condi-

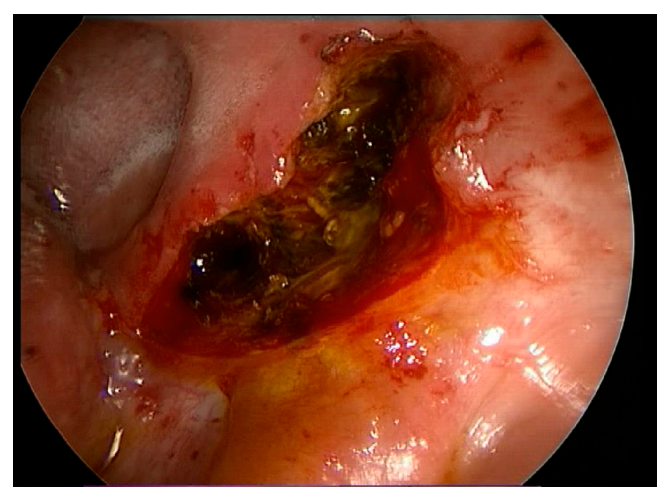

Figure 3. Surgical marsupialization. 


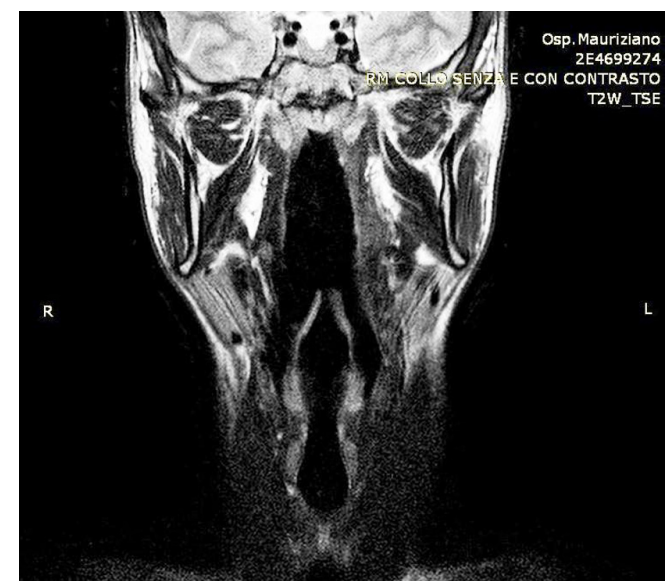

Figure 4. Radiological follow-up at 25 days after surgery showed no evidence of recurrence or sequelae.

tion should be considered in patients with a previous history of tonsillectomy, even if elective and apparently completely recovered.

\section{Conflict of Interest}

None.

\section{References}

1. Page C, Biet A, Zaatar R, Strunski V. Parapharyngeal abscess: diagnosis and treatment. Eur Arch Otorhinolaryngol. 2008;265(6):681-686.

2. Thapar A, Tassone P, Bhat N, Pfleiderer A. Parapharyngeal abscess: a life-threatening complication of quinsy. Clin Anat. 2008;21(1):23-26.

3. Monobe H, Suzuki S, Nakashima M, Tojima H, Kaga K. Peritonsillar abscess with parapharyngeal and retropharyngeal involvement: incidence and intraoral approach. Acta Otolaryngol Suppl. 2007;(559):91-94.

4. Sichel JY, Dano I, Hocwald E, Biron A, Eliashar R. Nonsurgical management of parapharyngeal space infections: a prospective study. Laryngoscope. 2002;112(5):906910.

5. McClay JE, Murray AD, Booth T. Intravenous antibiotic therapy for deep neck abscesses defined by computed tomography. Arch Otolaryngol Head Neck Surg. 2003;129(11):1207-1212.

6. Collin J, Beasley N. Tonsillitis to mediastinitis. J Laryngol Otol. 2006;120(11):963-966.

7. Koivunen P, Lopponen H. Internal carotid artery thrombosis and Horner's syndrome as complications of parapharyngeal abscess. Otolaryngol Head Neck Surg. 1999;121(1):160-162. 